\title{
Circular RNAs in the Regulation of Oxidative Stress
}

\author{
Yao Zhang ${ }^{1,2 t}$, Yu Chen ${ }^{1,2 \dagger}$, Yue Wan ${ }^{3 \dagger}$, Yueshui Zhao ${ }^{1,2}$, Qinglian Wen ${ }^{3}$, Xiaolong Tang ${ }^{1,2}$, \\ Jing Shen ${ }^{1,2}, X_{u} W^{1,2}$, Mingxing $L i^{1,2}$, Xiang $L i^{1}$, Jing $L i^{4}$, Wanping $L i^{1 *}$, Zhangang Xiao ${ }^{1,2 *}$ \\ and Fukuan $\mathrm{Du}^{1,2 *}$ \\ ${ }^{1}$ Laboratory of Molecular Pharmacology, Department of Pharmacology, School of Pharmacy, Southwest Medical University, \\ Luzhou, China, ${ }^{2}$ South Sichuan Institute of Translational Medicine, Luzhou, China, ${ }^{3}$ Department of Oncology, Affiliated Hospital of \\ Southwest Medical University, Luzhou, China, ${ }^{4}$ Department of Oncology and Hematology, Hospital (T.C.M) Affiliated to \\ Southwest Medical University, Luzhou, China
}

Oxidative stress caused by an imbalance between the production and elimination of reactive metabolites and free radicals can lead to the development of a variety of diseases.

OPEN ACCESS

Edited by:

Albert $P$ Li,

In Vitro ADMET Laboratories, United States

Reviewed by: Alberto Mantovani, National Institute of Health (ISS), Italy Ge Shan, University of Science and Technology of China, China

*Correspondence: Wanping $\mathrm{Li}$ 675832860@qq.com Zhangang Xiao xzg555898@hotmail.com

Fukuan Du

adublg@126.com

${ }^{+}$These authors have contributed equally to this work

Specialty section:

This article was submitted to Predictive Toxicology, a section of the journal Frontiers in Pharmacology

Received: 21 April 2021 Accepted: 13 July 2021 Published: 27 July 2021

Citation:

Zhang $Y$, Chen $Y$, Wan $Y$, Zhao $Y$, Wen $Q$, Tang $X$, Shen J, Wu X, Li M, Li X, Li J, Li W, Xiao Z and Du F (2021)

Circular RNAs in the Regulation of Oxidative Stress.

Front. Pharmacol. 12:697903. doi: 10.3389/fphar.2021.697903
Over the past years, with the development of science and technology, circular RNA (circRNA) has been found to be closely associated with oxidative stress, which plays an important role in the process of oxidative stress. Currently, the understanding of circRNAs in the mechanism of oxidative stress is limited. In this review, we described the relationship between oxidative stress and circRNAs, the circRNAs related to oxidative stress, and the role of circRNAs in promoting or inhibiting the occurrence and development of diseases associated with the oxidative stress system.

Keywords: circRNA, oxidative stress, ROS, free radicals, biomarker

\section{INTRODUCTION}

Oxidative stress is caused by an imbalance between the production of reactive oxygen species (ROS) and the antioxidant capacity (Birben et al., 2012). It is a negative effect produced by free radicals in the body. Persistent oxidative stress leads to the accumulation of ROS, destroying macromolecular substances, leading to DNA damage and further mutation, and induction of cancer and other diseases (Schieber and Chandel, 2014; Pisoschi and Pop, 2015; Baek and Lee, 2016) (Figure 1). Studies have found that oxidative stress is caused by a variety of factors and is regulated in many ways. As the main regulator" of antioxidant reactions, Nrf2 regulates the expression of genes involved in oxidative stress. Nrf2 protects cells from oxidative damage by inducing the expression of antioxidant and detoxification enzymes, thus activating the oxidative stress defense system in vivo (Hybertson et al., 2011; Ryoo and Kwak, 2018). However, Nrf2 cannot effectively scavenge the free radicals (Wang X. et al., 2019). This is due to oxidative stress drives the cellular physiological regulation response to be highly complicated, it is mainly achieved through signal transduction, transcription factors (TFs), and non-coding RNAs (ncRNAs) (Lin, 2019).

With the advent of advanced sequencing technologies, thousands of circRNAs have been identified in eukaryotes. Most circRNAs are predominantly derived from protein-coding genes and consist of one or more exons (Kristensen et al., 2019). These RNAs exhibit a covalently closed loop structure and do not contain the $5^{\prime}$ cap and $3^{\prime}$ poly (A) tail (Yu et al., 2019). Many studies have focused on the use of circRNAs as potential biomarkers for disease. Increasing number of studies are reporting that circRNAs are closely related to oxidative stress (Zhang J. et al., 2019; Chen et al., 2019; Yu et al., 2019; Hanan et al., 2020b; Saaoud et al., 2021), indicating that circRNA may play a central 


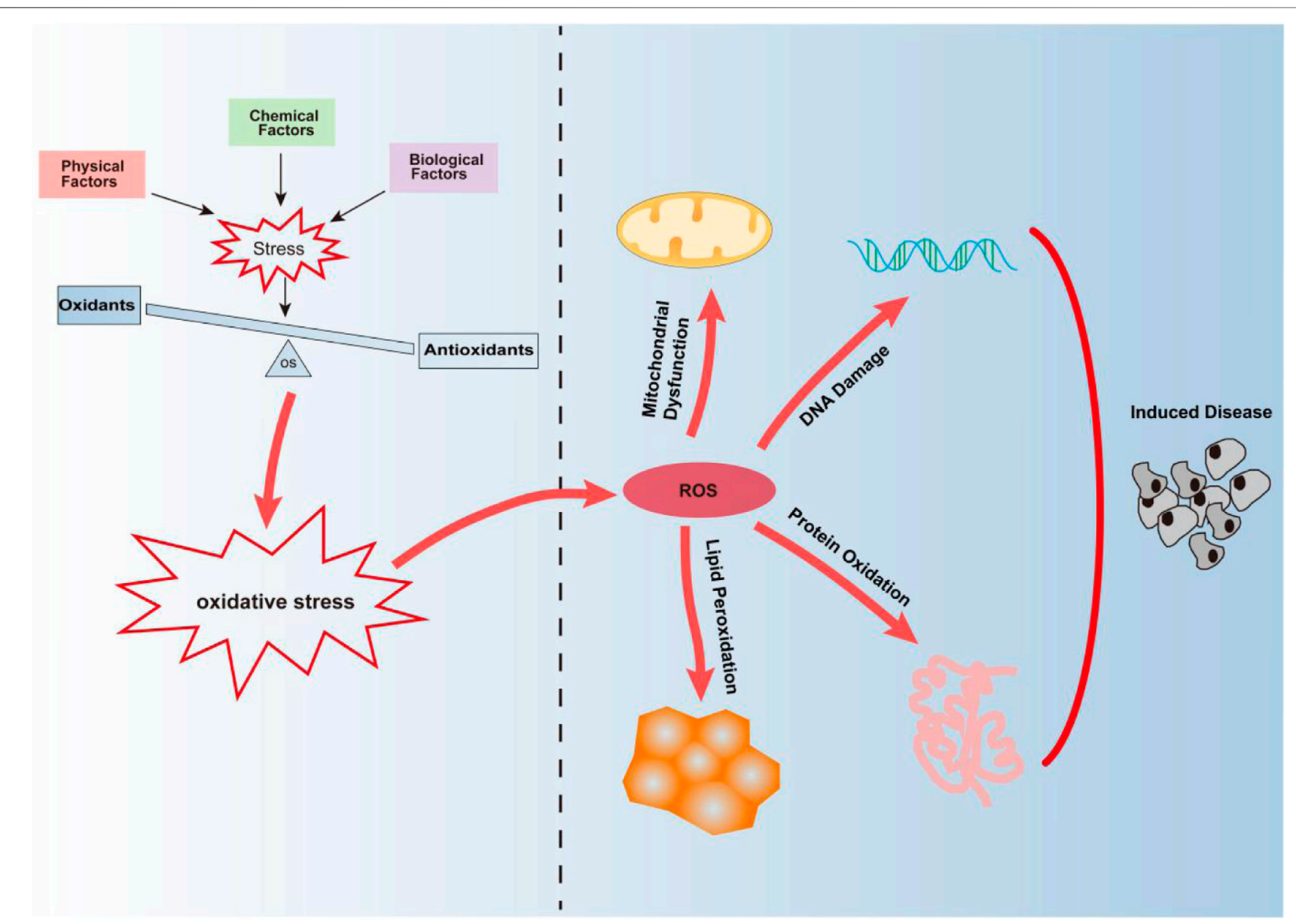

FIGURE 1 | Oxidative stress is mainly caused by the imbalance between oxidation and antioxidant defense mechanisms caused by physical, chemical, and biological factors. Continuous oxidative stress can lead to the accumulation of ROS leading to mitochondrial dysfunction, DNA damage, protein oxidation, and lipid peroxidation, thus inducing the occurrence of diseases.

role in the generation of oxidative stress, and that there is an urgent need to obtain a detailed understanding of this relationship.

Here, we mainly used the electronic database (PubMed) to conduct a literature review on the role of circRNAs with respect to the promotion or inhibition of the occurrence and development of oxidative-stress-related diseases. To this end, we used the following search terms in English: circRNA, circular RNA, noncoding RNA, oxidative stress, reactive oxygen species, ROS, free radicals, hypoxia, temperature, ionizing radiation, ultraviolet radiation, heavy metals, environmental toxins, medicine, nutrition, inflammation, and endoplasmic reticulum stress. There were no restrictions on the publication date. We reviewed the role of circRNAs in the generation of oxidative stress. Most importantly, we reviewed the current research progress on circRNA and oxidative-stress-related diseases and described the potential mechanisms underlying the generation of oxidative stress to enable the discovery of potential biomarkers related to the disease.

\section{FACTORS AFFECTING OXIDATIVE STRESS}

\section{Physical Factors}

Physical factors that induce oxidative stress include hypoxia, temperature, ionizing radiations, and ultraviolet rays. An imbalance between oxygen supply and demand can result in the induction of hypoxia (Semenza, 2001). Oxygen is the ultimate acceptor of high-energy electrons produced during different metabolic processes. Under physiological conditions, a small part of the oxygen that is not reduced during aerobic metabolism gives rise to ROS (Torres-Cuevas et al., 2017). Hypoxic conditions are conducive to the increase in ROS levels and generation of oxidative stress and induce the accumulation of ROS, a phenomenon that leads to oxidative damage (Quinonez-Flores et al., 2016). Oxidative stress that occurs during intermittent or continuous cellular hypoxia may be related to the development of hypertension, cardiovascular and metabolic disorders, and respiratory diseases. The imbalance between ROS generation and clearance is the core pathological 
mechanism associated with hypoxia (Pialoux and Mounier, 2012). Temperature is one of the causative factors of oxidative stress; oxidative damage can be induced when the body exposed to either continuous high temperatures or low temperatures (Venditti et al., 2010; Blagojevic et al., 2011; Rhoads et al., 2013; Klarod et al., 2015; Xue et al., 2018). Cells exposed to ionizing radiation are prone to oxidative stress. Chemical and biological changes occur in cells upon irradiation with ionizing radiation or in response to the radiolysis of cellular water (Azzam et al., 2012). These changes are not just restricted to the cells exposed to the ionizing radiations but instead also affect their offspring (Mothersill and Seymour, 2004; Spitz et al., 2004; Prise and O'Sullivan, 2009). The progeny of the bystander cells also exhibit perturbations in oxidative metabolism and extensive oxidative damage, including phenomena, such as protein carbonylation, lipid peroxidation, spontaneous gene mutations, and increased tumor transformation (Buonanno et al., 2011). Appropriate irradiation with ultraviolet rays can result in the induction of vitamin $\mathrm{D}$ synthesis, but long-term exposure to ultraviolet light can result in the development of skin cancer, malignant melanoma, and other diseases. ROS produced by ultraviolet radiation represents one of the mechanisms through which ultraviolet light exerts its harmful effects on human health (De Jager et al., 2017). The skin is highly susceptible to continuous ultraviolet radiation, which stimulates melanin synthesis and leads to pigmentation, epidermal melanocytes are susceptible to high ROS levels, induced in response to excessive sunlight. If the increase in ROS levels is sufficient to disrupt homeostasis, malignant transformation might occur (Picardo et al., 1996; Denat et al., 2014).

\section{Chemical Factors}

Chemicals present in the environment, drugs, food, and other substances can cause oxidative stress in the human body through certain mechanisms, which in turn trigger the development of oxidative-stress-related diseases. Oxidative stress is known to be responsible for more than 200 diseases, including bacterial, viral, and parasitic infections, autoimmune diseases, malignant tumors, atherosclerosis, diabetes, kidney diseases, skin diseases, and neurodegeneration (Pisoschi and Pop, 2015; Baek and Lee, 2016). Pesticides, dioxins, paraquat, quinones, heavy metals, PM2.5, and other toxic compounds in the environment can cause problems with the physiological response of the body and further damage the biological macromolecules, leading to the excessive accumulation of ROS and subsequent oxidative stress (Ahmad, 1995; Yoshida and Ogawa, 2000; Valko et al., 2005; Kukongviriyapan et al., 2016; Guo et al., 2017; WiniarskaMieczan, 2018). Drugs induce an increase in ROS levels, which in turn triggers the generation of oxidative stress. For example, glucocorticoids can indirectly induce oxidative stress by consuming antioxidant molecules or inhibiting the activity of antioxidant enzymes (Bjelakovic et al., 2007; Almeida et al., 2011; Feng and Tang, 2014). Cyclosporin A induces mitochondrial $\mathrm{Ca}^{2+}$ levels, increases oxidative stress and ROS production, and inhibits mitochondrial glucose metabolism and ATP production (Serkova et al., 2004). Insufficient or excessive nutrient intake can also lead to the generation of oxidative stress, and low intake or impaired availability of dietary antioxidants can weaken the antioxidant system (Sies et al., 2005; Saha et al., 2017).

\section{Biological Factors}

Biological factors contribute substantially to oxidative stress. The accumulation of ROS, inflammation, and endoplasmic reticulum (ER) stress is the main biological factors that contribute to oxidative stress. Mitochondria are the main sites of oxygen metabolism, and ROS are the by-products of oxygen consumption and cell metabolism (Zorov et al., 2014). ROS are oxygen-containing derivatives comprising extremely unstable free radicals (Dores et al., 1990). Under normal physiological conditions, the intracellular level of ROS is stably maintained to prevent cell damage. Non-enzymatic molecules or antioxidant enzymes can specifically promote the elimination of ROS (Liou and Storz, 2010). The increase in ROS content leads to the destruction of DNA, proteins, and lipids, resulting in the generation of oxidative stress.

During inflammation, mast cells and leukocytes are recruited to the injury site, resulting in the induction of a "respiratory burst". Increased oxygen uptake at the injury site leads to increased production and accumulation of ROS (Coussens and Werb, 2002). Inflammation and oxidative stress are closely linked (Rivera et al., 2017), and inflammation can result in the generation of a hypoxic microenvironment and induction of mitochondrial dysfunction, thereby triggering oxidative damage. Hypoxia promotes inflammation by activating HIF-1 and $\mathrm{NF}-\kappa \mathrm{B}$, and inflammatory cells release a large amount of ROS, thereby causing excessive oxidative damage (Mcgarry et al., 2018). Studies have shown that changes in the redox homeostasis of the ER are sufficient to induce ER stress, which in turn can stimulate the production of ROS in the ER and mitochondria (Cao and Kaufman, 2014). Reports have revealed a correlation between ER stress and oxidative stress (Zhang and Kaufman, 2008).

\section{THE EFFECT OF REACTIVE OXYGEN SPECIES ON CELL PHYSIOLOGY}

ROS are produced by oxidases and eliminated by a scavenging system, which comprises enzymatic or non-enzymatic reactions. An imbalance between the generation and removal of ROS results in increased ROS levels (Sun et al., 2020). Continuously elevated levels of ROS promote the development of cardiovascular diseases, various cancers, and neurodegenerative diseases (Yan et al., 2013; Sabharwal and Schumacker, 2014; Panth et al., 2016; Moloney and Cotter, 2018; Senoner and Dichtl, 2019). Under physiological conditions, the balance between ROS generation and clearance is stringently controlled (Zorov et al., 2014). Excessive ROS levels can result in reduced energy metabolism, deregulation of signal transduction and cell cycle, mutations in transport-related genes-and by extension, reduced biological activity-immune cell activation, and inflammation (Newsholme et al., 2016). ROS at appropriate concentrations plays an important regulatory and intermediary role in signal transduction, thereby protecting cells from oxidative stress and 
restoring "redox homeostasis." In higher organisms, ROS also function as signaling molecules to enable physiological functions, such as regulation of vascular tension and production of erythropoietin; they serve as ligands for various membrane receptors in various physiological processes (Droge, 2002).

ROS are key regulators of intracellular signaling pathways (Finkel, 2011). The process of ROS production by the NADPH oxidase 2 (NOX2) complex of phagocytes is referred to as "respiratory burst". It has always been thought that ROS are pro-inflammatory in nature. However, increasing numbers of studies indicate that ROS produced by the NOX2 complexes exert anti-inflammatory effects. Therefore, the production of ROS by phagocytes is considered an important part of innate immunity (Hultqvist et al., 2009; Sareila et al., 2011; Li et al., 2016). The ER and mitochondria are the main sources of ROS, and play a crucial role in the regulation of apoptosis and autophagy (Kaminskyy and Zhivotovsky, 2014). The use of antioxidants to interfere with the physiological functions of ROS may result in adverse effects. $\mathrm{Nrf} 2$ is the main transcription factor that regulates oxidative stress. It protects cells from oxidative damage by inducing the expression of antioxidant and detoxifying enzymes, thereby activating the antioxidant defense system of the body (Ryoo and Kwak, 2018). However, overexpression of Nrf2 results in increased expression of several molecules involved in intracellular redox balance maintenance, phase II detoxification, and cellular translocation, all of which can provide cancer cells with a growth advantage and induce the development of resistance against chemotherapy (Lau et al., 2008). Nrf2 activation in cancer cells not only leads to the development of drug resistance but also promotes their proliferation (Ohta et al., 2008). We can leverage the physiological properties of ROS to treat diseases. For example, targeting cancer cells through ROS-mediated mechanisms represents a new direction for cancer treatment in the future (Trachootham et al., 2009).

\section{CIRCRNA REGULATES REACTIVE OXYGEN SPECIES PRODUCTION}

ROS function as signaling molecules that cause physiological responses (Dupre-Crochet et al., 2013). Excessive ROS can induce oxidative stress, damage macromolecules, cause inflammation, and induce the accumulation of genetic mutations. Previous studies have reported a relationship between non-coding RNAs and oxidative stress. MicroRNAs might serve as highly effective biomarkers and therapeutic targets for oxidative-stressrelated diseases (Banerjee et al., 2017). Oxidative-stress-related long non-coding (lncRNAs) - as potential biomarkers and drug targets-may enable the development of new strategies for disease diagnosis and treatment (Wang X. et al., 2019). Compared with microRNAs and lncRNAs, circRNAs are more stable and resistant to exonuclease degradation. Further, as they function as sponges to regulate the effects of microRNAs (Saaoud et al., 2021), circRNAs can also play an important role in mediating the generation of oxidative stress. The expression of circRNAs is regulated by oxidative stress. They mediate the production of ROS and promote ROS-induced cell death, apoptosis, and inflammation (Saaoud et al., 2021). Studies have shown that L02 cells exhibit substantially increased circRNA-4099 levels in response to hydrogen peroxide $\left(\mathrm{H}_{2} \mathrm{O}_{2}\right)$-induced oxidative stress, and the generated circRNA-4099 further triggers the activation of the keap1/Nrf2-p38 MAPK cascade by enhancing the phosphorylation of related proteins, downregulating miR-706 expression, and enhancing the intracellular $\mathrm{H}_{2} \mathrm{O}_{2}$ levels, thereby promoting apoptosis, ROS generation, and fibrosis ( $\mathrm{Li}$ et al., 2020b). The circPRKCI-miR-545/589-E2F7 axis plays an important role in mediating $\mathrm{H}_{2} \mathrm{O}_{2}$-induced neuronal damage. $\mathrm{H}_{2} \mathrm{O}_{2}$ downregulates the expression of circPRKCI in neuronal cells, leading to miR-545/589 accumulation and downregulation of E2F7 expression. Heterotopic overexpression of circPRKCI, or transfection with miR-545/589 inhibitor, can effectively mitigate $\mathrm{H}_{2} \mathrm{O}_{2}$-induced neuronal death and apoptosis (Cheng et al., 2019).

\section{RESEARCH PROGRESS ON CIRCRNAS AND OXIDATIVE-STRESS-RELATED DISEASES}

The development of high-throughput sequencing technology has enabled scientists to discover thousands of circRNAs, which play a key role in biological processes (Hansen et al., 2013; Thomas and Saetrom, 2014). In addition to their miRNA-sponging functions, circRNAs are involved in phenomena, such as transcription, splicing, and translation, additionally, they also serve as protein decoys. (Legnini et al., 2017; Panda et al., 2017). CircRNAs can be divided into the following categories: exon circRNAs (ecircRNAs), circular intron RNA (ciRNA), exonintron circRNA (EIciRNA), and tRNA intron circRNAs (tricRNAs) based on sequence composition. Further, circRNAs can also be categorized as cytoplasmic and nuclear circRNAs based on their localization in the cell (Chen et al., 2015; Li et al., 2015; Zhao et al., 2019). As circRNAs can be categorized into different subtypes, their possible mechanisms of action are highly complicated. For example, the function as a transcriptional regulator may be unique to EIciRNA, but might not be necessarily extrapolated to other circRNAs (Chen et al., 2015), this makes circRNAs promising disease biomarkers.

Recently, it has been reported that circRNAs play a role in promoting or inhibiting the occurrence and development of diseases related to oxidative stress, such as cardiovascular diseases and Parkinson's disease (Li et al., 2018; Hanan et al., 2020a). Under physiological conditions, ROS affect the vascular function by regulating various redox-sensitive signaling pathways (Saaoud et al., 2021). Oxidative stress triggers the development of cardiovascular diseases by inducing vascular endothelial dysfunction and inflammation (Steven et al., 2019). Some studies have shown that the expression of some circRNAs is regulated by oxidative stress, and that these circRNAs mediate the production of ROS, and promote ROS-induced cell death and inflammation (Saaoud et al., 2021). Previous studies have reported that the expression of cZNF609 is significantly upregulated in vivo and in vitro in response to high glucose and hypoxia stress. Silencing of cZNF609 results in reduced loss of retinal blood vessels and inhibition of pathological 


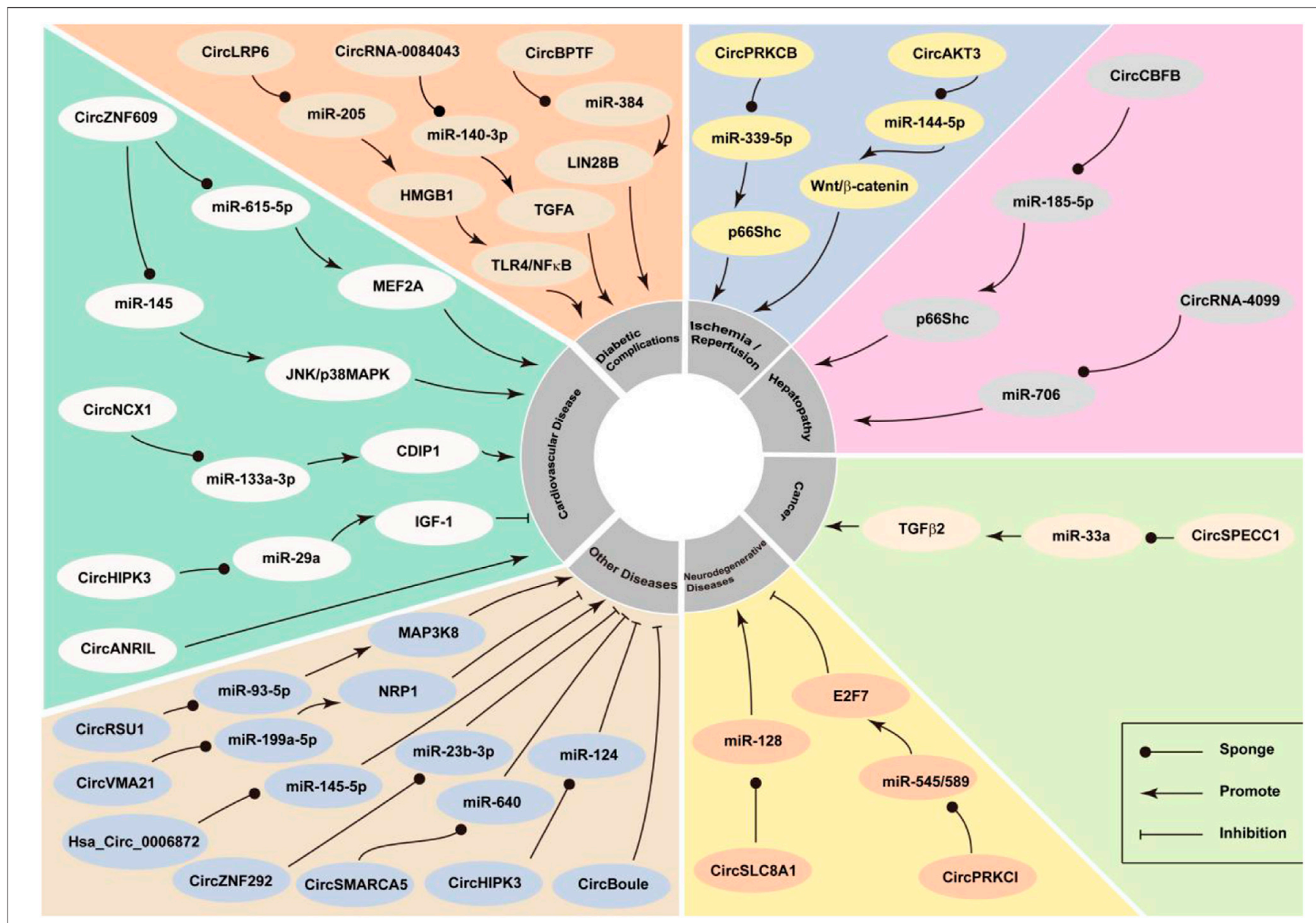

FIGURE 2 | CircRNA participates in oxidative stress and has the effect of increased oxidative stress or anti-oxidation, leading to the development or inhibition of oxidative stress-related diseases.

angiogenesis in vivo. Further, cZNF609 silencing results in increased endothelial cell migration and tube formation and protection of endothelial cells from oxidative and hypoxic stresses in vitro (Liu et al., 2017). CircHIPK3 is known to regulate lipopolysaccharide (LPS)-induced oxidative stress and inflammation in vivo and in vitro, upregulation of this circRNA is known to protect against LPS-induced oxidative damage and myocardial inflammation, and inhibit apoptosis (Fan et al., 2020). CircHIPK3 is a circular RNA whose expression is known to be significantly upregulated in hypoxic exosomes. This RNA is known to reduce oxidative stress-induced dysfunction of cardiac microvascular endothelial cells (CMVECs) via the miR-29a/IGF-1 axis (Wang Y. et al., 2019).

However, CircNFIX is another circular RNA whose expression is known to be significantly downregulated in cardiomyocytes in response to oxidative stress; this RNA could serve as a proapoptotic factor during cardiomyocyte apoptosis. Thus, circNFIX has the potential to serve as a biomarker and therapeutic target for myocardial infarction (Cui et al., 2020). CircHIPK3 is known to be significantly downregulated in response to $\mathrm{H}_{2} \mathrm{O}_{2}$-induced oxidative stress in OB-6 cells and primary human osteoblasts. Overexpression of circHIPK3 alleviates the $\mathrm{H}_{2} \mathrm{O}_{2}$-induced cell death (Liang J. et al., 2020). High glucose (HG) induces the downregulation of circACR in RSC96 cells. CircACR may downregulate the expression of miR$145-3 \mathrm{p}$ and promote the activation of the PI3K/AKT/mTOR pathway to alleviate HG-induced apoptosis, autophagy, and ROS generation in RSC96 cells (Liu et al., 2019). Heat stress may enhance the generation of oxidative stress (Slimen et al., 2014). Studies have revealed that circBoule regulates the expression of heat shock proteins under conditions of heat stress to protect male fertility in animals (Figure 2) (Gao et al., 2020). These studies indicate that circRNAs play an important role in the development of oxidative-stress-related diseases and that they can be used as a potential target for disease diagnosis and treatment.

\section{CIRCRNAS MEDIATE THE GENERATION OF OXIDATIVE STRESS}

Studies have reported that circRNAs play a role in the development of oxidative-stress-related diseases, and that is possibly mediated via the interaction between circRNAs and 
TABLE 1 | CircRNA regulates oxidative stress.

\begin{tabular}{|c|c|c|c|}
\hline CircRNA & Target & Function & References \\
\hline CircZNF609 & miR-615-5p; miR-145 & Promote oxidative stress & Liu et al. (2017), Ge and Gao (2020) \\
\hline CircLRP6 & miR-205 & Promote oxidative stress & Chen et al. (2019) \\
\hline CircNCX1 & miR-133a-3p & Promote oxidative stress & Ruan et al. (2020) \\
\hline CircSLC8A1 & $\mathrm{miR}-128$ & Oxidative stress-induced Parkinson's disease is a major participant & Hanan et al. (2020b) \\
\hline CircHIPK3 & miR-29a; miR-124 & Antioxidant & Wang et al. (2019b), Liang et al. (2020a) \\
\hline CircPRKCB & $\operatorname{miR}-339-5 p$ & Induced ROS accumulation & Feng et al. (2020) \\
\hline CircSMARCA5 & miR-640 & Inhibit oxidative stress & Cai et al. (2020) \\
\hline CircRNA_0084043 & miR-140-3p & Promote oxidative stress & Li et al. (2020a) \\
\hline CircANRIL & & Promote oxidative stress & Shi et al. (2020a) \\
\hline CircBPTF & miR-384 & Promote oxidative stress & Zhang and Sui (2020) \\
\hline CircAKT3 & $\operatorname{miR}-144-5 p$ & Promote oxidative stress & Xu et al. (2020) \\
\hline CircSPECC1 & miR-33a & Promote oxidative stress & Zhang et al. (2020a) \\
\hline Hsa_circ_0006872 & $\operatorname{miR}-145-5 p$ & Promote oxidative stress & Xue et al. (2021) \\
\hline CircVMA21 & miR-199a-5p; miR-9-3p & Inhibit oxidative stress & Shi et al. (2020b), Li et al. (2021) \\
\hline CircPRKCl & miR-545; miR-589 & Inhibit oxidative stress & Cheng et al. (2019) \\
\hline CircRSU1 & miR-93-5p & Promote oxidative stress & Yang et al. (2021) \\
\hline CircCBFB & $\operatorname{miR}-185-5 p$ & A key mediator of oxidative stress & Wang et al. (2020) \\
\hline CircRNA-4099 & miR-706 & Promote oxidative stress & Li et al. (2020b) \\
\hline CircZNF292 & miR-23b-3p & Regulate antioxidant genes & Liang et al. (2020b) \\
\hline
\end{tabular}

the key genes of the oxidative stress system (Figure 2). CircRNAs related to oxidative stress are summarised in Table $\mathbf{1}$.

\section{CircZNF609}

CircZNF609 originates as the result of the circularization of the second exon of the host gene. A 753-nucleotide open reading frame exists in circZNF609, i.e., from the AUG of the host gene to the stop codon generated by $3 \mathrm{nt}$ after splicing (Legnini et al., 2017). It has been reported that circZNF609 is an important intermediary for the generation of oxidative stress (Ge and Gao, 2020). CircZNF609 silencing may protect $\mathrm{HaCaT}$ cells from $\mathrm{H}_{2} \mathrm{O}_{2}$-induced oxidative stress via the regulation of the miR145-triggered JNK and p38MAPK signaling pathways ( $\mathrm{Ge}$ and Gao, 2020). There is evidence that circZNF609 acts as an endogenous sponge for miR-615-5p thereby resulting in MEF2A expression. Overexpression of MEF2A can rescue the effects of circZNF609 silencing on endothelial cell migration, tube formation, and apoptosis. Silencing of circZNF609 can result in the protection of endothelial cells from oxidative and hypoxic stresses and can be used as a potential strategy for the treatment of oxidative-stress-related vascular diseases (Liu et al., 2017).

\section{CircLRP6}

CircLRP6 is upregulated in mesangial cells treated with HG and acts as a molecular sponge for miR-205 to regulate HG-induced mesangial cell damage. In addition, overexpression of miR-205 or knockdown of circLRP6 results in the inhibition of the NF- $\kappa B$ pathway. As an intermediary of the newly discovered circLRP6miR-205-HMGB1-TLR4/NF- $\mathrm{BB}$ regulatory network, circLRP6 regulates HG-induced mesangial cell proliferation, oxidative stress, extracellular matrix accumulation, and inflammation by the following mechanisms: sponging miR-205, upregulating HMGB1, and activating the TLR4/NF- $\kappa B$ pathway (Chen et al., 2019). circLRP6 can serve as an important therapeutic biomarker for diabetic nephropathy.

\section{CircNCX1}

CircNCX1 is transcribed from the gene coding for sodium/calcium exchange protein 1 (NCX1). This RNA acts as a sponge for miR133a-3p, thereby inhibiting miR-133a-3p-mediated regulation of CDIP1 during myocardial ischemia-reperfusion (MI/R) injury and promoting cardiomyocyte apoptosis (Ruan et al., 2020). circNCX1 is highly expressed in cardiomyocytes. A study revealed a new regulatory pathway, involving circNCX1, miR-133a-3p, and CDIP1, all of which are related to cardiomyocyte apoptosis. This pathway can be used as a potential therapeutic target for ischemic heart disease (Li et al., 2018).

\section{CircSLC8A1}

CircSLC8A1 comprises an 1,832-basepair exon of the gene encoding solute carrier family 8 member 1 (SLC8A1), which is predominantly expressed in the cytoplasm of eukaryotic cells. As this RNA harbors multiple miRNA-binding sites (Dezfulian et al., 1987), it is related to cancer ( $\mathrm{Lu}$ et al., 2019; Zhu et al., 2021), cardiovascular disease (Lim et al., 2019; Tian et al., 2021), and Parkinson's disease (Hanan et al., 2020b). Some studies have also revealed that circSLC8A1 plays a role in the generation of oxidative stress (Hanan et al., 2020b). SH-SY cells (human neuronal cell line) exposed to paraquat-induced oxidative stress exhibited increased expression of circSLC8A1. However, cells treated with the neuroprotective antioxidant modifier, simvastatin, were characterized by decreased expression of circSLC8A1. Oxidation per se may result in the increased cyclization of circSLC8A1 or decreased degradation of circRNA in neurons. Therefore, we can speculate that circSLC8A1 is an important molecule in Parkinson's disease (Hanan et al., 2020b).

\section{CircHIPK3}

CircHIPK3 is a highly expressed stable RNA that is derived from exon 2 of HIPK3. This RNA was reported to bind to nine miRNAs that among themselves harbored 18 potential binding sites (Zheng et al., 2016). CircHIPK3 is known to be involved in 
the development and progression of cancer (Zeng et al., 2018; Zhang Y. et al., 2019). It can serve as a novel therapeutic target and diagnostic and prognostic biomarker in different cancer types (Zhang Y. et al., 2020). Recently, there has been a new understanding regarding the role of circHIPK3 as a potential target of oxidative stress. It has been demonstrated that overexpression of circHIPK3 significantly reduced apoptosis and the oxidative status in CMVECs exposed to oxidative stress. CircHIPK3 modulates oxidative damage in CMVEC via the miR-29a/IGF-1 axis. Studies demonstrated that circHIPK3 has an antioxidant function and is a potential target for protecting CMVECs from oxidative stress (Wang Y. et al., 2019). Several studies have investigated the expression and potential function of circHIPK 3 in the background of $\mathrm{H}_{2} \mathrm{O}_{2}$-induced oxidative damage in human osteoblasts. Downregulation of miR-124 expression by circHIPK3 may mediate $\mathrm{H}_{2} \mathrm{O}_{2}$-induced toxicity in human osteoblasts. Targeting the circHIPK3-miR-124 cascade serves as a strategy to protect human osteoblasts from oxidative damage (Liang J. et al., 2020).

\section{CircPRKCB}

p66Shc plays a key role in ROS production (Napoli et al., 2003). CircPRKCB promotes the accumulation of ROS in response to intestinal I/R injury by sponging miR-339-5p and upregulating the expression of p66Shc. The key role of the circPRKCB/miR$339-5 \mathrm{p} / \mathrm{p} 66 \mathrm{Shc}$ signaling pathway in regulating $\mathrm{I} / \mathrm{R}$ intestinal oxidative stress has been demonstrated. This pathway may serve as a potential therapeutic target for intestinal $I / R$ injury (Feng et al., 2020).

\section{CircSMARCA5}

CircSMARCA5 (also known as hsa_circ_0001445) originates from exons 15 and 16 of SMARCA5, which encodes a specific chromatin remodeling protein $\mathrm{SNF} 2 \mathrm{H}$ that regulates chromatin structure to promote DNA transcription and repair (Cai et al., 2019). Overexpression of hsa_circ_0001445 can inhibit the inflammatory response of oxidized low-density lipoproteintreated human umbilical vein endothelial cells and reduces the expression of inflammatory factors and the level of oxidative stress (Cai et al., 2020).

\section{CircRNA 0084043}

The deletion of circRNA_0084043 protects against HG-induced ARPE-19 cell damage by sponging miR-140-3p to induce the expression of transforming growth factor-alpha. The deletion of circRNA_0084043 significantly improved the cell survival rate and inhibited HG-induced apoptosis. In addition, circRNA_0084043 knockout significantly reduced oxidative stress, decreased the malondialdehyde content, and increased superoxide dismutase and glutathione peroxidase activities. Silencing of circRNA_0084043 effectively inhibited the HGinduced inflammatory response; this might serve as a potential therapeutic strategy for diabetic retinopathy (Li et al., 2020a).

\section{CircANRIL}

CircANRIL is a circular antisense non-coding RNA transcribed from the INK4 locus (atherosclerotic cardiovascular disease locus) on chromosome 9p21 (Holdt et al., 2016). High expression of circANRIL in the human vascular tissue is known to be related to reduced severity of coronary artery disease (CAD). Inhibition of circANRIL expression in CAD can reduce vascular endothelial damage, oxidative stress, and inflammation, and can therefore serve as a new diagnostic and treatment strategy (Shi P. et al., 2020).

\section{CircBPTF}

CircBPTF (hsa_circ_0000799) is a novel circRNA that is spliced by reverse splicing from exons 21 to 27 of the gene encoding bromodomain PHD finger transcription factor (BPTF) (Bi et al., 2018). Knockout of circBPTF can improve the activity of human umbilical vein endothelial cells (HUVECs) and inhibit HGinduced apoptosis, inflammatory response, and oxidative stress. In conclusion, CircBPTF knockout acts via the miR384/Lin28b axis in HUVECs, which has a protective effect on HG-induced inflammatory damage and oxidative stress, thereby providing guidance for future treatment strategies for diabetesrelated vascular complications (Zhang and Sui, 2020).

\section{CircAKT3}

CircRNA AKT3 (circAKT3) is a novel RNA derived from AKT3 (Zang et al., 2021), It mediates renal I/R (RI/R) damage by regulating oxidative stress and the miR-144-5p/Wnt/ $\beta$-catenin pathway. CircAKT3 can promote apoptosis and activate the Wnt/ $\beta$-catenin signaling pathway, thereby serving as a new target for the treatment of RI/R injury (Xu et al., 2020).

\section{CircSPECC1}

CircSPECC1 (hsa_circ_0000745) is a novel circRNA, which has been reported to be significantly downregulated in $\mathrm{H}_{2} \mathrm{O}_{2}$-treated HCC cells. Knocking out circSPECC1 in the background under $\mathrm{H}_{2} \mathrm{O}_{2}$ treatment, inhibited the proliferation of HCC cells and promoted their apoptosis. In addition, circSPECC1 inhibits miR33a expression through direct interaction, and miR-33a inhibitors can partially reverse the effects of circSPECC1 knockdown on proliferation and apoptosis. CircSPECC1 acts as a miR-33a sponge, regulating TGF $\beta-2$ and autophagy under conditions of oxidative stress to promote the occurrence of HCC tumors. These findings may provide potential treatment strategies for patients with liver cancer (Zhang B. et al., 2020).

\section{Hsa_circ_0006872}

Hsa_circ_0006872 levels were increased in patients with chronic obstructive pulmonary disease and negatively correlated with miR-145-5p levels. Hsa_circ_0006872 promotes apoptosis, inflammation, and oxidative stress triggered by cigarette smoke extract in HPMECs and BEAS-2B cells by regulating the miR-145-5p/NF- $\kappa$ B pathway. Hsa_circ_0006872 silencing inhibits CSE-induced cell damage by regulating miR-145-5p (Xue et al., 2021).

\section{CircVMA21}

CircVMA21 (also known as hsa_circ_0091702) is derived from the gene encoding vacuolar ATPase assembly factor (VMA21) (Cheng et al., 2018). Studies have found that circVMA21 can 
decrease apoptosis in rats to reduce intervertebral disc degeneration and protect WI-38 cells from LPS-induced damage. Overexpression of circVMA21 inhibited LPS-induced, apoptosis, inflammation, and oxidative stress in THP-1 cells. The results showed that circVMA21 regulates the expression of NRP1 by sponging miR-199a-5p (Li et al., 2021). CircVMA21 plays an important role in affecting acute renal lesions related to sepsis by regulating the miR-9-3p/SMG1/inflammatory axis and oxidative stress (Shi Y. et al., 2020).

\section{CircPRKCI}

CircPRKCI, which is a 1,484 bp long circRNA, is spliced in the opposite direction from the two exons (15 and 16) of PRKCI located at 3q26.2 and plays a key role in the occurrence and development of a variety of digestive system tumors (Zhang X. et al., 2019; Qi et al., 2019). In cultured SH-SY5Y nerve cells, $\mathrm{H}_{2} \mathrm{O}_{2}$ downregulated the expression of circPRKCI, leading to the accumulation of miR-545 and miR-589, but the expression of its target transcription factor E2F7 decreased. Importantly, ectopic overexpression of circPRKCI attenuated $\mathrm{H}_{2} \mathrm{O}_{2}$-induced cytotoxicity. The results of this study showed that the imbalance in the PRKCI-miR-545/589-E2F7 axis mediates $\mathrm{H}_{2} \mathrm{O}_{2}$-induced neuronal damage. Targeting this new cascade may be a new strategy for protecting neurons against oxidative damage (Cheng et al., 2019).

\section{CircRSU1}

CircRSU1 was significantly upregulated in human articular chondrocytes and osteoarthritis (OA) articular cartilage treated with $\mathrm{H}_{2} \mathrm{O}_{2}$. CircRSU1 expression is induced by IL- $1 \beta$ and $\mathrm{H}_{2} \mathrm{O}_{2}$ stimulation and subsequently occurs through the MEK/ERK1/2 and NF- $\kappa B$ pathways. Studies have shown that the circRSU1-miR-93$5 \mathrm{p}-\mathrm{MAP} 3 \mathrm{~K} 8$ axis plays a critical role in the regulation of circRSU1 in oxidative stress and ECM homeostasis in human chondrocytes. This axis affects OA through oxidative stress regulation and can be used as a potential target for OA treatment (Yang et al., 2021).

\section{CircCBFB}

CircCBFB is a circRNA derived from the core-binding factor-beta $(C B F B)$ gene locus and is highly expressed in acetaminophen (APAP) treatment. p66Shc is the main regulator of mitochondrial ROS and a key mediator of oxidative stress in liver cells. CircCBFB acts as a miR-185-5p sponge and regulates APAP-induced liver damage by targeting p66Shc. This provides a potential therapeutic target for APAP-induced liver damage (Wang et al., 2020).

\section{CircRNA-4099}

CircRNA-4099 (hsa_circRNA_100759) is located in exon 11 (Wang et al., 2018). Hepatic fibers are always induced by oxidative stress. $\mathrm{H}_{2} \mathrm{O}_{2}$ induces the $\mathrm{L} 02$ cell line to inhibit viability and promote cell apoptosis, ROS production, and cell fibrosis, as well as the keap1/Nrf2 and p38MAPK cascades. $\mathrm{H}_{2} \mathrm{O}_{2}$ stimulated circRNA-4099, and an increased level of circRNA4099 enhanced $\mathrm{H}_{2} \mathrm{O}_{2}$-induced damage by inhibiting miR-706. CircRNA-4099 inhibits miR-706 expression by triggering the keap1/Nrf2 and p38MAPK pathways in L02 cells, aggravating $\mathrm{H}_{2} \mathrm{O}_{2}$-induced damage, and provides a promising therapeutic target for hepatitis or hepatic fibrosis (Li et al., 2020b).

\section{CircZNF292}

Oxidative stress is one of the key factors in the pathogenesis of agerelated cataracts (ARC) (Berthoud and Beyer, 2009). The level of circZNF292 decreased significantly and that of miR-23b-3p increased significantly in ARC. CircZNF292 acts as a competitive endogenous RNA and regulates the expression of antioxidant genes by competing with miR-23b-3p. The results of this study showed that circZNF292, a circRNA which is downregulated in the anterior lens capsule of patients with ARC, can act as a miR-23b-3p sponge and participate in the resistance to oxidative damage and anti-apoptosis of lens epithelial cells, thereby serving as a potential biomarker for the prevention and treatment of ARC (Liang S. et al., 2020).

\section{CONCLUSION}

In recent years, scientists have become increasingly enthusiastic about circRNA research. Nonetheless, the mechanisms of action of circRNAs have still not been completely elucidated. In this review, we focused on circRNAs related to oxidative stress, introduced their mechanisms of action, and provided potential therapeutic targets for oxidative-stressrelated diseases, but problems still exist. First, with respect to the mechanism of action of circRNA in oxidative-stress-related diseases, most studies have focused on the circRNA-miRNA-mRNA regulatory networks, but there is very little research on the interaction between circRNAs. Future research needs to systematically study the mechanism of action of circRNA in the context of oxidative-stress-related diseases to clarify the relationship between circRNA and oxidative-stress-related diseases. In addition, because circRNA is categorized into multiple subtypes, different functional mechanisms are possible. In future studies, the roles of different subtypes of circRNAs and oxidativestress-related diseases should be emphasized. Finally, as promising biomarkers and drug targets, circRNAs may provide a new direction for the diagnosis and treatment of diseases. Future research should focus on the precise mechanism of action of circRNA with respect to the regulation of oxidative stress.

\section{AUTHOR CONTRIBUTIONS}

All authors contributed to the conception and design, writing, critical revision, and final approval of the article. YaZ, YuZ and QW conceived the article, YC and YW revised this article, WL, ZX and FD supervised the study and coordinated the writing of the manuscript. All authors have read and agreed to the published version of the manuscript.

\section{FUNDING}

This work was supported by the Strategic Cooperation Project for Transfer and Transformation of Scientific and Technological achievements of Southwest Medical University and Lu County Government (grant no. 2019LXXNYKD-07), Joint Founds of Southwest Medical University and Luzhou Government (No.2020LZXNYDJ08), Funds of talent introduction and scientific research of Southwest Medical University (No.05-40140). 


\section{REFERENCES}

Ahmad, S. (1995). Oxidative Stress from Environmental Pollutants. Arch. Insect Biochem. Physiol. 29, 135-157. doi:10.1002/arch.940290205

Almeida, M., Han, L., Ambrogini, E., Weinstein, R. S., and Manolagas, S. C. (2011). Glucocorticoids and Tumor Necrosis Factor a Increase Oxidative Stress and Suppress Wnt Protein Signaling in Osteoblasts. J. Biol. Chem. 286, 44326-44335. doi:10.1074/jbc.m111.283481

Azzam, E. I., Jay-Gerin, J.-P., and Pain, D. (2012). Ionizing Radiation-Induced Metabolic Oxidative Stress and Prolonged Cell Injury. Cancer Lett. 327, 48-60. doi:10.1016/j.canlet.2011.12.012

Baek, J., and Lee, M.-G. (2016). Oxidative Stress and Antioxidant Strategies in Dermatology. Redox Rep. 21, 164-169. doi:10.1179/1351000215y.0000000015

Banerjee, J., Khanna, S., and Bhattacharya, A. (2017). MicroRNA Regulation of Oxidative Stress. Oxid Med. Cel Longev 2017, 2872156. doi:10.1155/2017/ 2872156

Berthoud, V. M., and Beyer, E. C. (2009). Oxidative Stress, Lens gap Junctions, and Cataracts. Antioxid. Redox Signaling 11, 339-353. doi:10.1089/ars.2008.2119

Bi, J., Liu, H., Cai, Z., Dong, W., Jiang, N., Yang, M., et al. (2018). Circ-BPTF Promotes Bladder Cancer Progression and Recurrence through the miR-31-5p/ RAB27A axis. Aging 10, 1964-1976. doi:10.18632/aging.101520

Birben, E., Sahiner, U. M., Sackesen, C., Erzurum, S., and Kalayci, O. (2012). Oxidative Stress and Antioxidant Defense. World Allergy Organ. J. 5, 9-19. doi:10.1097/wox.0b013e3182439613

Bjelakovic, G., Beninati, S., Pavlovic, D., Kocic, G., Jevtovic, T., Kamenov, B., et al. (2007). Glucocorticoids and Oxidative Stress. J. Basic Clin. Physiol. Pharmacol. $18,115-127$

Blagojevic, D. P., Grubor-Lajsic, G. N., and Spasic, M. B. (2011). Cold Defence Responses the Role of Oxidative Stress. Front. Biosci. S3, 416-427. doi:10.2741/ s161

Buonanno, M., De Toledo, S. M., and Azzam, E. I. (2011). Increased Frequency of Spontaneous Neoplastic Transformation in Progeny of Bystander Cells from Cultures Exposed to Densely Ionizing Radiation. PLoS One 6, e21540. doi:10.1371/journal.pone.0021540

Cai, J., Chen, Z., and Zuo, X. (2019). circSMARCA5 Functions as a Diagnostic and Prognostic Biomarker for Gastric Cancer. Dis. Markers 2019, 2473652. doi:10.1155/2019/9356804

Cai, Y., Xu, L., Xu, C., Wang, Y., and Fan, C. (2020). Hsa_circ_0001445 Inhibits Ox-LDL-Induced HUVECs Inflammation, Oxidative Stress and Apoptosis by Regulating miRNA-640. Perfusion, 267659120979472. doi:10.1177/ 0267659120979472

Cao, S. S., and Kaufman, R. J. (2014). Endoplasmic Reticulum Stress and Oxidative Stress in Cell Fate Decision and Human Disease. Antioxid. Redox Signaling 21, 396-413. doi:10.1089/ars.2014.5851

Chen, B., Li, Y., Liu, Y., and Xu, Z. (2019). circLRP6 Regulates High Glucoseinduced Proliferation, Oxidative Stress, ECM Accumulation, and Inflammation in Mesangial Cells. J. Cel Physiol 234, 21249-21259. doi:10.1002/jcp. 28730

Chen, L., Huang, C., Wang, X., and Shan, G. (2015). Circular RNAs in Eukaryotic Cells. Cg 16, 312-318. doi:10.2174/1389202916666150707161554

Cheng, Q., Cao, X., Xue, L., Xia, L., and Xu, Y. (2019). CircPRKCI-miR-545/589E2F7 axis Dysregulation Mediates Hydrogen Peroxide-Induced Neuronal Cell Injury. Biochem. Biophysical Res. Commun. 514, 428-435. doi:10.1016/ j.bbrc.2019.04.131

Cheng, X., Zhang, L., Zhang, K., Zhang, G., Hu, Y., Sun, X., et al. (2018). Circular RNA VMA21 Protects against Intervertebral Disc Degeneration through Targeting miR-200c and X Linked Inhibitor-Of-Apoptosis Protein. Ann. Rheum. Dis. 77, 770-779. doi:10.1136/annrheumdis-2017-212056

Coussens, L. M., and Werb, Z. (2002). Inflammation and Cancer. Nature 420, 860-867. doi:10.1038/nature01322

Cui, X., Dong, Y., Li, M., Wang, X., Jiang, M., Yang, W., et al. (2020). A Circular RNA from NFIX Facilitates Oxidative Stress-Induced H9c2 Cells Apoptosis. In Vitro Cell.Dev.Biol.-Animal 56, 715-722. doi:10.1007/s11626-020-00476-Z

De Jager, T. L., Cockrell, A. E., and Du Plessis, S. S. (2017). Ultraviolet Light Induced Generation of Reactive Oxygen Species. Adv. Exp. Med. Biol. 996, 15-23. doi:10.1007/978-3-319-56017-5_2
Denat, L., Kadekaro, A. L., Marrot, L., Leachman, S. A., and Abdel-Malek, Z. A. (2014). Melanocytes as Instigators and Victims of Oxidative Stress. J. Invest. Dermatol. 134, 1512-1518. doi:10.1038/jid.2014.65

Dezfulian, M., Bitar, R. A., and Bartlett, J. G. (1987). Kinetics Study of Immunological Response to Clostridium Botulinum Toxin. J. Clin. Microbiol. 25, 1336-1337. doi:10.1128/jcm.25.7.1336-1337.1987

Dores, R. M., Mcdonald, L. K., Steveson, T. C., and Sei, C. A. (1990). The Molecular Evolution of Neuropeptides: Prospects for the '90s. Brain Behav. Evol. 36, 80-99. doi:10.1159/000115300

Dröge, W. (2002). Free Radicals in the Physiological Control of Cell Function. Physiol. Rev. 82, 47-95. doi:10.1152/physrev.00018.2001

Dupré-Crochet, S., Erard, M., and Nüße, O. (2013). ROS Production in Phagocytes: Why, when, and where?. J. Leukoc. Biol. 94, 657-670. doi:10.1189/jlb.1012544

Fan, S., Hu, K., Zhang, D., and Liu, F. (2020). Interference of circRNA HIPK3 Alleviates Cardiac Dysfunction in Lipopolysaccharide-Induced Mice Models and Apoptosis in H9C2 Cardiomyocytes. Ann. Transl Med. 8, 1147. doi:10.21037/atm-20-5306

Feng, D., Wang, Z., Zhao, Y., Li, Y., Liu, D., Chen, Z., et al. (2020). Circ-PRKCB Acts as a ceRNA to Regulate p66Shc-Mediated Oxidative Stress in Intestinal Ischemia/reperfusion. Theranostics 10, 10680-10696. doi:10.7150/thno.44250

Feng, Y.-L., and Tang, X.-L. (2014). Effect of Glucocorticoid-Induced Oxidative Stress on the Expression of Cbfa1. Chemico-Biological Interactions 207, 26-31. doi:10.1016/j.cbi.2013.11.004

Finkel, T. (2011). Signal Transduction by Reactive Oxygen Species. J. Cel Biol 194, 7-15. doi:10.1083/jcb.201102095

Gao, L., Chang, S., Xia, W., Wang, X., Zhang, C., Cheng, L., et al. (2020). Circular RNAs from BOULE Play Conserved Roles in protection against Stress-Induced Fertility Decline. Sci. Adv. 6. doi:10.1126/sciadv.abb7426

Ge, R., and Gao, G. (2020). Anti-antioxidant Impacts of circZNF609 Silence in HaCaT Cells through Regulating miR-145. Artif. Cell Nanomedicine, Biotechnol. 48, 384-392. doi:10.1080/21691401.2019.1709863

Guo, Z., Hong, Z., Dong, W., Deng, C., Zhao, R., Xu, J., et al. (2017). PM2.5Induced Oxidative Stress and Mitochondrial Damage in the Nasal Mucosa of Rats. Int. J. Environ. Res. Public Health 14 (2), 134. doi:10.3390/ijerph14020134

Hanan, M., Simchovitz, A., Yayon, N., Vaknine, S., Cohen-Fultheim, R., Karmon, M., et al. (2020a). A Parkinson's Disease CircRNAs Resource Reveals a Link between circSLC8A1 and Oxidative Stress. EMBO Mol. Med. 12, e13551. doi:10.15252/emmm.202013551

Hanan, M., Simchovitz, A., Yayon, N., Vaknine, S., Cohen-Fultheim, R., Karmon, M., et al. (2020b). A Parkinson's Disease CircRNAs Resource Reveals a Link between circSLC8A1 and Oxidative Stress. EMBO Mol. Med. 12, e11942. doi:10.15252/emmm.202013551

Hansen, T. B., Jensen, T. I., Clausen, B. H., Bramsen, J. B., Finsen, B., Damgaard, C. K., et al. (2013). Natural RNA Circles Function as Efficient microRNA Sponges. Nature 495, 384-388. doi:10.1038/nature11993

Holdt, L. M., Stahringer, A., Sass, K., Pichler, G., Kulak, N. A., Wilfert, W., et al. (2016). Circular Non-coding RNA ANRIL Modulates Ribosomal RNA Maturation and Atherosclerosis in Humans. Nat. Commun. 7, 12429. doi:10.1038/ncomms12429

Hultqvist, M., Olsson, L. M., Gelderman, K. A., and Holmdahl, R. (2009). The Protective Role of ROS in Autoimmune Disease. Trends Immunol. 30, 201-208. doi:10.1016/j.it.2009.03.004

Hybertson, B. M., Gao, B., Bose, S. K., and Mccord, J. M. (2011). Oxidative Stress in Health and Disease: the Therapeutic Potential of Nrf2 Activation. Mol. Aspects Med. 32, 234-246. doi:10.1016/j.mam.2011.10.006

Kaminskyy, V. O., and Zhivotovsky, B. (2014). Free Radicals in Cross Talk between Autophagy and Apoptosis. Antioxid. Redox Signaling 21, 86-102. doi:10.1089/ ars.2013.5746

Klarod, K., Gatterer, H., Frontull, V., Philippe, M., and Burtscher, M. (2015). Effects of Short-Term Antioxidant Supplementation on Oxidative Stress and Exercise Performance in the Heat and the Cold. Int. J. Physiol. Pathophysiol Pharmacol. 7, 98-104.

Kristensen, L. S., Andersen, M. S., Stagsted, L. V. W., Ebbesen, K. K., Hansen, T. B., and Kjems, J. (2019). The Biogenesis, Biology and Characterization of Circular RNAs. Nat. Rev. Genet. 20, 675-691. doi:10.1038/s41576-019-0158-7

Kukongviriyapan, U., Apaijit, K., and Kukongviriyapan, V. (2016). Oxidative Stress and Cardiovascular Dysfunction Associated with Cadmium Exposure: 
Beneficial Effects of Curcumin and Tetrahydrocurcumin. Tohoku J. Exp. Med. 239, 25-38. doi:10.1620/tjem.239.25

Lau, A., Villeneuve, N., Sun, Z., Wong, P., and Zhang, D. (2008). Dual Roles of Nrf2 in Cancer. Pharmacol. Res. 58, 262-270. doi:10.1016/j.phrs.2008.09.003

Legnini, I., Di Timoteo, G., Rossi, F., Morlando, M., Briganti, F., Sthandier, O., et al. (2017). Circ-ZNF609 Is a Circular RNA that Can Be Translated and Functions in Myogenesis. Mol. Cel 66, 22-37. doi:10.1016/j.molcel.2017.02.017

Li, M., Ding, W., Tariq, M. A., Chang, W., Zhang, X., Xu, W., et al. (2018). A Circular Transcript of Ncxl Gene Mediates Ischemic Myocardial Injury by Targeting miR-133a-3p. Theranostics 8, 5855-5869. doi:10.7150/thno.27285

Li, R., Jia, Z., and Trush, M. A. (2016). Defining ROS in Biology and Medicine. React. Oxyg Species (Apex) 1, 9-21. doi:10.20455/ros.2016.803

Li, X., Li, R., Gong, Q., Shi, D., Song, L., and Song, Y. (2021). Circular RNA circVMA21 Ameliorates Lipopolysaccharide (LPS)-induced Acute Kidney Injury by Targeting the miR-199a-5p/NRP1 axis in Sepsis. Biochem. Biophysical Res. Commun. 548, 174-181. doi:10.1016/j.bbrc.2021.02.028

Li, Y., Cheng, T., Wan, C., and Cang, Y. (2020a). circRNA_0084043 Contributes to the Progression of Diabetic Retinopathy via Sponging miR-140-3p and Inducing TGFA Gene Expression in Retinal Pigment Epithelial Cells. Gene 747, 144653. doi:10.1016/j.gene.2020.144653

Li, Y., Gao, X., Wang, Z., Liu, W., Xu, F., Hu, Y., et al. (2020b). Circular RNA 4099 Aggravates Hydrogen Peroxide-Induced Injury by Down-Regulating microRNA-706 in L02 Cells. Life Sci. 241, 116826. doi:10.1016/j.lfs.2019.116826

Li, Z., Huang, C., Bao, C., Chen, L., Lin, M., Wang, X., et al. (2015). Exon-intron Circular RNAs Regulate Transcription in the Nucleus. Nat. Struct. Mol. Biol. 22, 256-264. doi:10.1038/nsmb.2959

Liang, J., Shen, Y.-c., Zhang, X.-y., Chen, C., Zhao, H., and Hu, J. (2020a). Circular RNA HIPK3 Downregulation Mediates Hydrogen Peroxide-Induced Cytotoxicity in Human Osteoblasts. Aging 12, 1159-1170. doi:10.18632/ aging. 102674

Liang, S., Dou, S., Li, W., and Huang, Y. (2020b). Profiling of Circular RNAs in Age-Related Cataract Reveals circZNF292 as an Antioxidant by Sponging miR23b-3p. Aging 12, 17271-17287. doi:10.18632/aging.103683

Lim, T. B., Aliwarga, E., Luu, T. D. A., Li, Y. P., Ng, S. L., Annadoray, L., et al. (2019). Targeting the Highly Abundant Circular RNA circSlc8al in Cardiomyocytes Attenuates Pressure Overload Induced Hypertrophy. Cardiovasc. Res. 115, 1998-2007. doi:10.1093/cvr/cvz130

Lin, Y. H. (2019). MicroRNA Networks Modulate Oxidative Stress in Cancer. Int. J. Mol. Sci. 20. doi:10.3390/ijms20184497

Liou, G.-Y., and Storz, P. (2010). Reactive Oxygen Species in Cancer. Free Radic. Res. 44, 479-496. doi:10.3109/10715761003667554

Liu, C., Yao, M.-D., Li, C.-P., Shan, K., Yang, H., Wang, J.-J., et al. (2017). Silencing of Circular RNA-Znf609 Ameliorates Vascular Endothelial Dysfunction. Theranostics 7, 2863-2877. doi:10.7150/thno.19353

Liu, Y., Chen, X., Yao, J., and Kang, J. (2019). Circular RNA ACR Relieves High Glucose-Aroused RSC96 Cell Apoptosis and Autophagy via Declining microRNA-145-3p. J. Cel Biochem. doi:10.1002/jcb.29568

Lu, Q., Liu, T., Feng, H., Yang, R., Zhao, X., Chen, W., et al. (2019). Circular RNA circSLC8A1 Acts as a Sponge of miR-130b/miR-494 in Suppressing Bladder Cancer Progression via Regulating PTEN. Mol. Cancer 18, 111. doi:10.1186/ s12943-019-1040-0

Mcgarry, T., Biniecka, M., Veale, D. J., and Fearon, U. (2018). Hypoxia, Oxidative Stress and Inflammation. Free Radic. Biol. Med. 125, 15-24. doi:10.1016/ j.freeradbiomed.2018.03.042

Moloney, J. N., and Cotter, T. G. (2018). ROS Signalling in the Biology of Cancer. Semin. Cel Develop. Biol. 80, 50-64. doi:10.1016/j.semcdb.2017.05.023

Mothersill, C., and Seymour, C. B. (2004). Radiation-induced Bystander EffectsIimplications for Cancer. Nat. Rev. Cancer 4, 158-164. doi:10.1038/nrc1277

Napoli, C., Martin-Padura, I., De Nigris, F., Giorgio, M., Mansueto, G., Somma, P., et al. (2003). Deletion of the p66Shc Longevity Gene Reduces Systemic and Tissue Oxidative Stress, Vascular Cell Apoptosis, and Early Atherogenesis in Mice Fed a High-Fat Diet. Proc. Natl. Acad. Sci. 100, 2112-2116. doi:10.1073/ pnas. 0336359100

Newsholme, P., Cruzat, V. F., Keane, K. N., Carlessi, R., and De Bittencourt, P. I. H., Jr. (2016). Molecular Mechanisms of ROS Production and Oxidative Stress in Diabetes. Biochem. J. 473, 4527-4550. doi:10.1042/bcj20160503c

Ohta, T., Iijima, K., Miyamoto, M., Nakahara, I., Tanaka, H., Ohtsuji, M., et al. (2008). Loss of Keap1 Function Activates Nrf2 and Provides Advantages for
Lung Cancer Cell Growth. Cancer Res. 68, 1303-1309. doi:10.1158/00085472.can-07-5003

Panda, A. C., Grammatikakis, I., Munk, R., Gorospe, M., and Abdelmohsen, K. (2017). Emerging Roles and Context of Circular RNAs. Wiley Interdiscip. Rev. RNA 8, el386. doi:10.1002/wrna.1386

Panth, N., Paudel, K. R., and Parajuli, K. (2016). Reactive Oxygen Species: A Key Hallmark of Cardiovascular Disease. Adv. Med. 2016, 9152732. doi:10.1155/ 2016/9152732

Pialoux, V., and Mounier, R. (2012). Hypoxia-induced Oxidative Stress in Health Disorders. Oxid Med. Cel Longev 2012, 940121. doi:10.1155/2012/940121

Picardo, M., Grammatico, P., Roccella, F., Roccella, M., Grandinetti, M., Del Porto, G., et al. (1996). Imbalance in the Antioxidant Pool in Melanoma Cells and normal Melanocytes from Patients with Melanoma. J. Invest. Dermatol. 107, 322-326. doi:10.1111/1523-1747.ep12363163

Pisoschi, A. M., and Pop, A. (2015). The Role of Antioxidants in the Chemistry of Oxidative Stress: A Review. Eur. J. Med. Chem. 97, 55-74. doi:10.1016/ j.ejmech.2015.04.040

Prise, K. M., and O'sullivan, J. M. (2009). Radiation-induced Bystander Signalling in Cancer Therapy. Nat. Rev. Cancer 9, 351-360. doi:10.1038/nrc2603

Qi, S.-X., Sun, H., Liu, H., Yu, J., Jiang, Z.-Y., and Yan, P. (2019). Role and Mechanism of Circ-PRKCI in Hepatocellular Carcinoma. Wjg 25, 1964-1974. doi:10.3748/wjg.v25.i16.1964

Quinonez-Flores, C. M., Gonzalez-Chavez, S. A., and Pacheco-Tena, C. (2016). Hypoxia and its Implications in Rheumatoid Arthritis. J. Biomed. Sci. 23, 62. doi:10.1186/s12929-016-0281-0

Rhoads, R. P., Baumgard, L. H., Suagee, J. K., and Sanders, S. R. (2013). Nutritional Interventions to Alleviate the Negative Consequences of Heat Stress. Adv. Nutr. 4, 267-276. doi:10.3945/an.112.003376

Rivera, J. C., Dabouz, R., Noueihed, B., Omri, S., Tahiri, H., and Chemtob, S. (2017). Ischemic Retinopathies: Oxidative Stress and Inflammation. Oxid Med. Cel Longev 2017, 3940241. doi:10.1155/2017/3940241

Ruan, Z., Yu, W., and Wang, S. (2020). CircNCX1/miR-133a: A Potential Novel Biomarker and Risk Factor Predictor for Myocardial Ischemia-Reperfusion Injury. Int. J. Cardiol. 299, 256. doi:10.1016/j.ijcard.2019.07.014

Ryoo, I.-g., and Kwak, M.-K. (2018). Regulatory Crosstalk between the Oxidative Stress-Related Transcription Factor Nfe2l2/Nrf2 and Mitochondria. Toxicol. Appl. Pharmacol. 359, 24-33. doi:10.1016/j.taap.2018.09.014

Saaoud, F., Drummer I.V., C., Shao, Y., Sun, Y., Lu, Y., Xu, K., et al. (2021). Circular RNAs Are a Novel Type of Non-coding RNAs in ROS Regulation, Cardiovascular Metabolic Inflammations and Cancers. Pharmacol. Ther. 220, 107715. doi:10.1016/j.pharmthera.2020.107715

Sabharwal, S. S., and Schumacker, P. T. (2014). Mitochondrial ROS in Cancer: Initiators, Amplifiers or an Achilles' Heel?. Nat. Rev. Cancer 14, 709-721. doi: $10.1038 / \mathrm{nrc} 3803$

Saha, S. K., Lee, S. B., Won, J., Choi, H. Y., Kim, K., Yang, G. M., et al. (2017). Correlation between Oxidative Stress, Nutrition, and Cancer Initiation. Int. J. Mol. Sci. 18. doi:10.3390/ijms18071544

Sareila, O., Kelkka, T., Pizzolla, A., Hultqvist, M., and Holmdahl, R. (2011). NOX2 Complex-Derived ROS as Immune Regulators. Antioxid. Redox Signaling 15, 2197-2208. doi:10.1089/ars.2010.3635

Schieber, M., and Chandel, N. S. (2014). ROS Function in Redox Signaling and Oxidative Stress. Curr. Biol. 24, R453-R462. doi:10.1016/j.cub.2014.03.034

Semenza, G. L. (2001). Hypoxia-inducible Factor 1: Oxygen Homeostasis and Disease Pathophysiology. Trends Mol. Med. 7, 345-350. doi:10.1016/s14714914(01)02090-1

Senoner, T., and Dichtl, W. (2019). Oxidative Stress in Cardiovascular Diseases: Still a Therapeutic Target?. Nutrients 11. doi:10.3390/nu11092090

Serkova, N. J., Christians, U., and Benet, L. Z. (2004). Biochemical Mechanisms of Cyclosporine Neurotoxicity. Mol. Interventions 4, 97-107. doi:10.1124/ mi.4.2.7

Shi, P., Ji, H., Zhang, H., Yang, J., Guo, R., and Wang, J. (2020a). circANRIL Reduces Vascular Endothelial Injury, Oxidative Stress and Inflammation in Rats with Coronary Atherosclerosis. Exp. Ther. Med. 20, 2245-2251. doi:10.3892/etm.2020.8956

Shi, Y., Sun, C. F., Ge, W. H., Du, Y. P., and Hu, N. B. (2020b). Circular RNA VMA21 Ameliorates Sepsis-associated Acute Kidney Injury by Regulating miR9-3p/SMG1/inflammation axis and Oxidative Stress. J. Cel. Mol. Med. 24, 11397-11408. doi:10.1111/jcmm.15741 
Sies, H., Stahl, W., and Sevanian, A. (2005). Nutritional, Dietary and Postprandial Oxidative Stress. J. Nutr. 135, 969-972. doi:10.1093/jn/135.5.969

Slimen, I. B., Najar, T., Ghram, A., Dabbebi, H., Ben Mrad, M., and Abdrabbah, M. (2014). Reactive Oxygen Species, Heat Stress and Oxidative-Induced Mitochondrial Damage. A Review. Int. J. Hyperthermia 30, 513-523. doi:10.3109/02656736.2014.971446

Spitz, D. R., Azzam, E. I., Jian Li, J., and Gius, D. (2004). Metabolic Oxidation/ reduction Reactions and Cellular Responses to Ionizing Radiation: a Unifying Concept in Stress Response Biology. Cancer Metastasis Rev. 23, 311-322. doi:10.1023/b:canc.0000031769.14728.bc

Steven, S., Frenis, K., Oelze, M., Kalinovic, S., Kuntic, M., Bayo Jimenez, M. T., et al. (2019). Vascular Inflammation and Oxidative Stress: Major Triggers for Cardiovascular Disease. Oxid Med. Cel Longev 2019, 7092151. doi:10.1155/ 2019/7092151

Sun, Y., Lu, Y., Saredy, J., Wang, X., Drummer Iv, C., Shao, Y., et al. (2020). ROS Systems Are a New Integrated Network for Sensing Homeostasis and Alarming Stresses in Organelle Metabolic Processes. Redox Biol. 37, 101696. doi:10.1016/ j.redox.2020.101696

Thomas, L. F., and Sætrom, P. (2014). Circular RNAs Are Depleted of Polymorphisms at microRNA Binding Sites. Bioinformatics 30, 2243-2246. doi:10.1093/bioinformatics/btu257

Tian, M., Xue, J., Dai, C., Jiang, E., Zhu, B., and Pang, H. (2021). CircSLC8A1 and circNFIX Can Be Used as Auxiliary Diagnostic Markers for Sudden Cardiac Death Caused by Acute Ischemic Heart Disease. Sci. Rep. 11, 4695. doi:10.1038/ s41598-021-84056-5

Torres-Cuevas, I., Parra-Llorca, A., Sánchez-Illana, A., Nuñez-Ramiro, A., Kuligowski, J., Cháfer-Pericás, C., et al. (2017). Oxygen and Oxidative Stress in the Perinatal Period. Redox Biol. 12, 674-681. doi:10.1016/j.redox.2017.03.011

Trachootham, D., Alexandre, J., and Huang, P. (2009). Targeting Cancer Cells by ROS-Mediated Mechanisms: a Radical Therapeutic Approach?. Nat. Rev. Drug Discov. 8, 579-591. doi:10.1038/nrd2803

Valko, M., Morris, H., and Cronin, M. (2005). Metals, Toxicity and Oxidative Stress. Cmc 12, 1161-1208. doi:10.2174/0929867053764635

Venditti, P., Di Stefano, L., and Di Meo, S. (2010). Oxidative Stress in ColdInduced Hyperthyroid State. J. Exp. Biol. 213, 2899-2911. doi:10.1242/ jeb.043307

Wang, H., He, P., Pan, H., Long, J., Wang, J., Li, Z., et al. (2018). Circular RNA Circ4099 Is Induced by TNF- $\alpha$ and Regulates ECM Synthesis by Blocking miR-6165p Inhibition of Sox9 in Intervertebral Disc Degeneration. Exp. Mol. Med. 50, 1-14. doi:10.1038/s12276-018-0056-7

Wang, X., Shen, C., Zhu, J., Shen, G., Li, Z., and Dong, J. (2019a). Long Noncoding RNAs in the Regulation of Oxidative Stress. Oxid Med. Cel Longev 2019, 1318795. doi:10.1155/2019/1318795

Wang, Y., Zhao, R., Liu, W., Wang, Z., Rong, J., Long, X., et al. (2019b). Exosomal circHIPK3 Released from Hypoxia-Pretreated Cardiomyocytes Regulates Oxidative Damage in Cardiac Microvascular Endothelial Cells via the miR29a/IGF-1 Pathway. Oxid Med. Cel Longev 2019, 7954657. doi:10.1155/2019/ 7954657

Wang, Z., Zhao, Y., Sun, R., Sun, Y., Liu, D., Lin, M., et al. (2020). Circ-CBFB Upregulates p66Shc to Perturb Mitochondrial Dynamics in APAP-Induced Liver Injury. Cell Death Dis 11, 953. doi:10.1038/s41419-020-03160-y

Winiarska-Mieczan, A. (2018). Protective Effect of tea against lead and CadmiumInduced Oxidative Stress-A Review. Biometals 31, 909-926. doi:10.1007/ s10534-018-0153-Z

Xu, Y., Jiang, W., Zhong, L., Li, H., Bai, L., Chen, X., et al. (2020). circ-AKT3 Aggravates Renal Ischaemia-Reperfusion Injury via Regulating miR-144-5p/ Wnt/beta-Catenin Pathway and Oxidative Stress. J. Cel Mol Med. doi:10.1111/ jcmm.16072

Xue, C., Lu, H., Liu, Y., Zhang, J., Wang, J., Luo, W., et al. (2018). Trans-Ferulic Acid-4-Beta-Glucoside Alleviates Cold-Induced Oxidative Stress and Promotes Cold Tolerance. Int. J. Mol. Sci. 19 (8), 2321. doi:10.3390/ijms19082321

Xue, M., Peng, N., Zhu, X., and Zhang, H. (2021). Hsa_circ_0006872 Promotes Cigarette Smoke-Induced Apoptosis, Inflammation and Oxidative Stress in HPMECs and BEAS-2B Cells through the miR-145-5p/NF-Kb axis. Biochem. Biophysical Res. Commun. 534, 553-560. doi:10.1016/j.bbrc.2020.11.044

Yan, M. H., Wang, X., and Zhu, X. (2013). Mitochondrial Defects and Oxidative Stress in Alzheimer Disease and Parkinson Disease. Free Radic. Biol. Med. 62, 90-101. doi:10.1016/j.freeradbiomed.2012.11.014
Yang, Y., Shen, P., Yao, T., Ma, J., Chen, Z., Zhu, J., et al. (2021). Novel Role of circRSU1 in the Progression of Osteoarthritis by Adjusting Oxidative Stress. Theranostics 11, 1877-1900. doi:10.7150/thno.53307

Yoshida, R., and Ogawa, Y. (2000). Oxidative Stress Induced by 2,3,7,8Tetrachlorodibenzo-P-Dioxin. An Application of Oxidative Stress Markers to Cancer Risk Assessment of Dioxins. Ind. Health 38, 5-14. doi:10.2486/ indhealth. 38.5

Yu, T., Wang, Y., Fan, Y., Fang, N., Wang, T., Xu, T., et al. (2019). CircRNAs in Cancer Metabolism: a Review. J. Hematol. Oncol. 12, 90. doi:10.1186/s13045-019-0776-8

Zang, H.-L., Ji, F.-J., Ju, H.-Y., and Tian, X.-F. (2021). Circular RNA AKT3 Governs Malignant Behaviors of Esophageal Cancer Cells by Sponging miR-17-5p. Wjg 27, 240-254. doi:10.3748/wjg.v27.i3.240

Zeng, K., Chen, X., Xu, M., Liu, X., Hu, X., Xu, T., et al. (2018). CircHIPK3 Promotes Colorectal Cancer Growth and Metastasis by Sponging miR-7. Cel Death Dis 9, 417. doi:10.1038/s41419-018-0454-8

Zhang, B., Liu, Z., Cao, K., Shan, W., Liu, J., Wen, Q., et al. (2020a). Circ-SPECC1 Modulates TGF $\beta 2$ and Autophagy under Oxidative Stress by Sponging miR-33a to Promote Hepatocellular Carcinoma Tumorigenesis. Cancer Med. 9, 5999-6008. doi:10.1002/cam4.3219

Zhang, J., Li, D., Wang, D., Man, K., and Yang, X. (2019a). CircRNA Expression Profiles in Human Dental Pulp Stromal Cells Undergoing Oxidative Stress. J. Transl Med. 17, 327. doi:10.1186/s12967-019-2078-x

Zhang, K., and Kaufman, R. J. (2008). From Endoplasmic-Reticulum Stress to the Inflammatory Response. Nature 454, 455-462. doi:10.1038/nature07203

Zhang, W., and Sui, Y. (2020). CircBPTF Knockdown Ameliorates High GlucoseInduced Inflammatory Injuries and Oxidative Stress by Targeting the miR-384/ LIN28B axis in Human Umbilical Vein Endothelial Cells. Mol. Cel Biochem 471, 101-111. doi:10.1007/s11010-020-03770-2

Zhang, X., Yang, H., Zhao, L., Li, G., and Duan, Y. (2019b). Circular RNA PRKCI Promotes Glioma Cell Progression by Inhibiting microRNA-545. Cel Death Dis 10, 616. doi:10.1038/s41419-019-1863-z

Zhang, Y., Liu, Q., and Liao, Q. (2020b). CircHIPK3: a Promising Cancer-Related Circular RNA. Am. J. Transl Res. 12, 6694-6704.

Zhang, Y., Li, C., Liu, X., Wang, Y., Zhao, R., Yang, Y., et al. (2019c). circHIPK3 Promotes Oxaliplatin-Resistance in Colorectal Cancer through Autophagy by Sponging miR-637. EBioMedicine 48, 277-288. doi:10.1016/j.ebiom.2019.09.051

Zhao, X., Cai, Y., and Xu, J. (2019). Circular RNAs: Biogenesis, Mechanism, and Function in Human Cancers. Int. J. Mol. Sci. 20 (16), 3926. doi:10.3390/ ijms 20163926

Zheng, Q., Bao, C., Guo, W., Li, S., Chen, J., Chen, B., et al. (2016). Circular RNA Profiling Reveals an Abundant circHIPK3 that Regulates Cell Growth by Sponging Multiple miRNAs. Nat. Commun. 7, 11215. doi:10.1038/ ncomms 11215

Zhu, Q., Zhang, X., Zai, H.-Y., Jiang, W., Zhang, K.-J., He, Y.-Q., et al. (2021). circSLC8A1 Sponges miR-671 to Regulate Breast Cancer Tumorigenesis via PTEN/PI3k/Akt Pathway. Genomics 113, 398-410. doi:10.1016/ j.ygeno.2020.12.006

Zorov, D. B., Juhaszova, M., and Sollott, S. J. (2014). Mitochondrial Reactive Oxygen Species (ROS) and ROS-Induced ROS Release. Physiol. Rev. 94, 909-950. doi:10.1152/physrev.00026.2013

Conflict of Interest: The authors declare that the research was conducted in the absence of any commercial or financial relationships that could be construed as a potential conflict of interest.

Publisher's Note: All claims expressed in this article are solely those of the authors and do not necessarily represent those of their affiliated organizations, or those of the publisher, the editors and the reviewers. Any product that may be evaluated in this article, or claim that may be made by its manufacturer, is not guaranteed or endorsed by the publisher.

Copyright $\odot 2021$ Zhang, Chen, Wan, Zhao, Wen, Tang, Shen, Wu, Li, $L i, L i, L i$, Xiao and $\mathrm{Du}$. This is an open-access article distributed under the terms of the Creative Commons Attribution License (CC BY). The use, distribution or reproduction in other forums is permitted, provided the original author(s) and the copyright owner(s) are credited and that the original publication in this journal is cited, in accordance with accepted academic practice. No use, distribution or reproduction is permitted which does not comply with these terms. 